Fifth International Conference on Sustainable Construction Materials and

Technologies. http://www.claisse.info/Proceedings.htm

\title{
STUDY ON VARIOUS FACTORS RELATED TO THE EVALUATION OF THERMAL CRACKING PROBABILITY OF MASS CONCRETE STRUCTURES
}

\author{
Ryoichi Ashizawa ${ }^{1}$, Toshiaki Mizobuchi ${ }^{2 a}$, and Hiroki Izumi ${ }^{2 b}$ \\ ${ }^{1}$ Concrete and Construction Materials Group, Kajima Technical Research Institute, \\ 2-19-1, Tobitakyu, Chofu-City, Tokyo Japan. ${ }^{1}$ Email: <ashizawa@ kajima.com> \\ ${ }^{2}$ Department of Civil and Environmental Engineering, Faculty of Engineering and \\ Design, HOSEI University, 3-7-2, Kajino, Koganei-City, Tokyo Japan. \\ ${ }^{2 a}$ Email: <mizobuch@hosei.ac.jp>, ${ }^{2 b}$ Email: < hiroki.izumi.4f@stu.hosei.ac.jp>
}

\begin{abstract}
To appropriately evaluate the thermal cracking probability, thermal stress analyses were conducted on multiple side walls constructed under the same conditions of concrete mix proportion and the same construction method. In addition, the thermal cracking rates were obtained from actual occurrences of thermal cracking, and the relationship between the thermal cracking index and thermal cracking probability was stochastically studied using the R-S model. The results indicated that the homogeneity of the structure might affect the thermal cracking probability and that the correction of the thermal cracking indexes might enable evaluation of the actual thermal cracking probability.
\end{abstract}

Keywords: mass concrete, thermal stress, thermal cracking probability, R-S model

\section{INTRODUCTION}

Thermal cracking has a significant effect on the durability and watertightness of concrete structures. Therefore, it is necessary to evaluate the risk of thermal cracking beforehand and control it by applying appropriate countermeasures. The ratio of the tensile strength and tensile stress (hereafter "thermal cracking index") of concrete can be used as an indicator for evaluating thermal cracking. Theoretically, when the tensile stress in concrete exceeds the tensile strength, that is, when the thermal cracking index is smaller than 1.0, thermal cracking occurs. Because tensile strength and tensile stress accompany variations, however, thermal cracking is evaluated using the relationship between the thermal cracking index and the thermal cracking probability, as shown in Figure 1, in the "Standard Specifications for Concrete Structures 2017" by the Japan Society of Civil Engineers (hereafter "JSCE Specifications") and "The Guidelines for Control of Cracking of Mass Concrete 2016" by the Japan Concrete Institute (hereafter 
"JCI Guidelines"). This figure relates the actual occurrences of thermal cracking in multiple different structures to the thermal cracking indexes obtained from thermal stress analyses using the three-dimensional finite element method.

In this study, thermal stress analyses were conducted on multiple side walls constructed at different placement period but under the same conditions of concrete mix proportion and the same construction method, and the rates of thermal cracking in the structures were obtained from the actual occurrences of thermal cracking. In addition, thermal cracking probabilities were stochastically obtained using the R-S model. By comparing both, the factors affecting the thermal cracking probability as well as their degree of influence were studied.

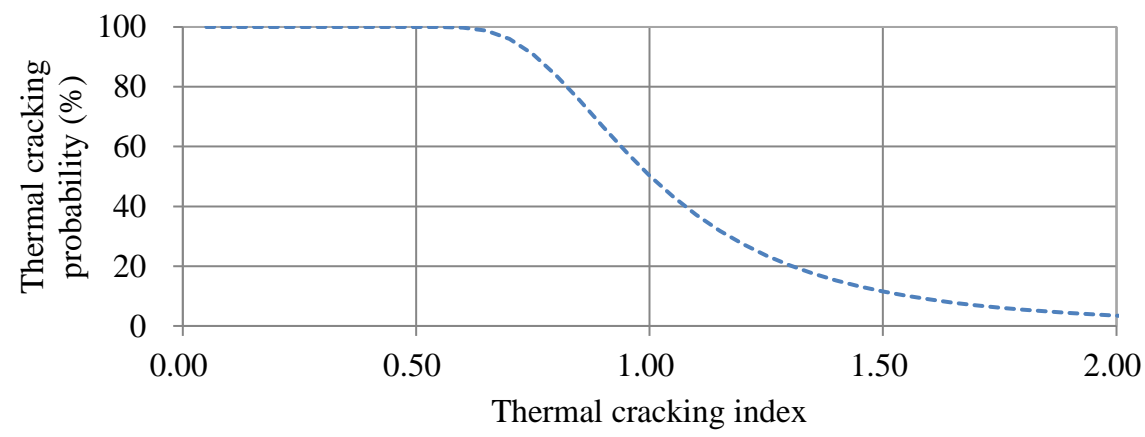

Figure 1. Relationship between thermal cracking indexes and thermal cracking probabilities as shown in the JCI Guideline

\section{OUTLINE OF TARGET STRUCTURES}

In this study, multiple side walls constructed in a single box culvert were evaluated. As Figure 2 shows, the side walls were $4.0-5.0 \mathrm{~m}$ in height, $1.1-2.2 \mathrm{~m}$ in thickness, and 5-20 $\mathrm{m}$ in length, and consisted of 58 members in total. Each side wall had crack control joints at intervals of $5.0 \mathrm{~m}$. The concrete was placed throughout the year, under the same conditions including the mix proportion and basic construction method.

Table 1 show the mix proportion. Portland blast furnace slag cement type B was used for the concrete, with a water-cement ratio of $50 \%$, and a retarding type air-entraining and water-reducing admixture was used during the summer (from June to October).

Placement and compaction of the concrete was performed by carefully following the JSCE Specifications. Steel forms were used for every side wall and the forms were removed about five days after placement of the concrete, and curing was conducted with a sheet covering until the material age was 14 days. The intervals between the concrete placement of the bottom slab and that of the side wall were about 40-280 days. 


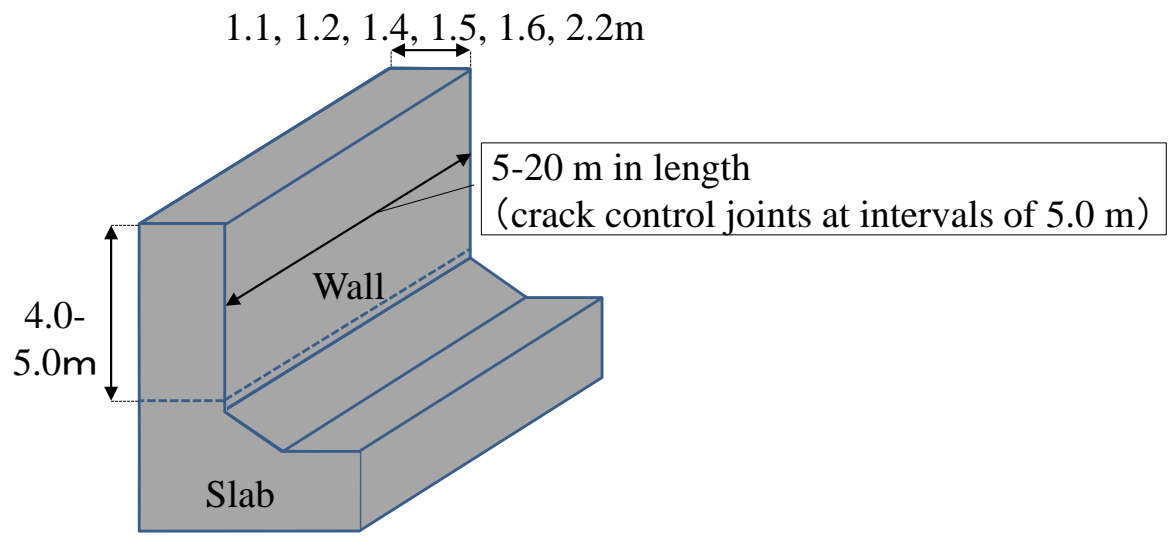

Figure 2. Outline of the sidewall

Table 1. Mix proportion of the concrete

\begin{tabular}{|c|c|c|c|c|c|c|c|c|c|}
\hline \multirow{2}{*}{$\begin{array}{l}\text { Gmax } \\
(\mathrm{mm})\end{array}$} & \multirow{2}{*}{$\begin{array}{l}\text { W/C } \\
(\%)\end{array}$} & \multirow{2}{*}{$\begin{array}{c}\text { Slump } \\
(\mathrm{cm})\end{array}$} & \multirow{2}{*}{$\begin{array}{l}\text { Air } \\
(\%)\end{array}$} & \multirow{2}{*}{$\begin{array}{l}\text { s/a } \\
(\%)\end{array}$} & \multicolumn{5}{|c|}{ Unit quantity $\left(\mathrm{kg} / \mathrm{m}^{3}\right)$} \\
\hline & & & & & $\mathrm{W}$ & $\mathrm{C}$ & $\mathrm{S}$ & $\mathrm{G}$ & Ad \\
\hline 20 & 50 & 8.0 & 4.5 & 42.0 & 150 & 300 & 783 & 1100 & 3.0 \\
\hline
\end{tabular}

Note; W; Tap water, C; Portland blast furnace slag cement type B $\left(3.04 \mathrm{~g} / \mathrm{cm}^{3}\right), \mathrm{S}$;

Fine aggregate $\left(2.63 \mathrm{~g} / \mathrm{cm}^{3}\right), \mathrm{G}$; Coarse aggregate $\left(2.69 \mathrm{~g} / \mathrm{cm}^{3}\right)$, Ad; Airentraining and water-reducing admixture

\section{EVALUATION METHOD}

\section{Thermal Cracking Index, Tensile Stress, and Tensile Strength}

Thermal stress analyses using the three-dimensional finite element method were conducted on the 58 members of the side wall. Half-symmetry models reflecting the size and shape of each side wall were used for the analysis model. For the analysis conditions, the actual construction conditions were reflected in the placing season of concrete, temperature at the time of placement, and strength development formula. The strength development formula was set for each side wall based on the formula shown in the JCI Guideline, from the results at the material age of 7 and 28 days (standard water curing) conducted as part of quality control during the actual construction. For other analysis conditions, the calculation formulas for the thermal properties, tensile strength, elastic modulus, etc. conformed to the JCI Guideline.

From the analysis results, the minimum thermal cracking indexes and the corresponding tensile stress and tensile strength were obtained.

\section{Rate of Thermal Cracking}

Assuming the part of the wall between the crack control joints at intervals of $5.0 \mathrm{~m}$ as one section and dividing the thermal cracking index gained from the analysis results above by 0.05 , the thermal cracking rates were obtained from the total number of 
sections within a division of thermal cracking index and the number of sections where thermal cracking actually occurred within the same division. The total number of sections was 130 .

\section{Thermal Cracking Probability}

The thermal cracking rates discussed above were obtained by relating the observation data on actual structures to the results of thermal stress analysis, and thus, are not based on a stochastic point of view that considers variations in material, construction, etc. As a stochastic method, the R-S model, which considers variations in tensile stress and tensile strength, has been suggested (Figure 3) [Nakamura et al. 1995], [Sugihashi, 2017]. This model assumes that both the tensile stress and tensile strength follow a normal distribution, and the probability that tensile stress exceeds tensile strength is calculated as the thermal cracking probability using equation (1). In this study, the coefficients of variation of the tensile stress and tensile strength obtained from the results of the thermal stress analysis have been used.

$$
\begin{gathered}
Z=\frac{-I c r+1}{\sqrt{V_{s}^{2} \cdot I c r^{2}+V_{c}^{2}}} \\
P(Z)=\frac{1}{2}\left\{1+\operatorname{erf}\left(\frac{Z}{\sqrt{2}}\right)\right\}
\end{gathered}
$$

where $Z$ is normal random variable, $P(Z)$ is the thermal cracking probability, Icr is the thermal cracking index, $V_{c}$ is the coefficient of variation of the tensile stress, and $V_{s}$ is the coefficient of variation of the tensile strength.

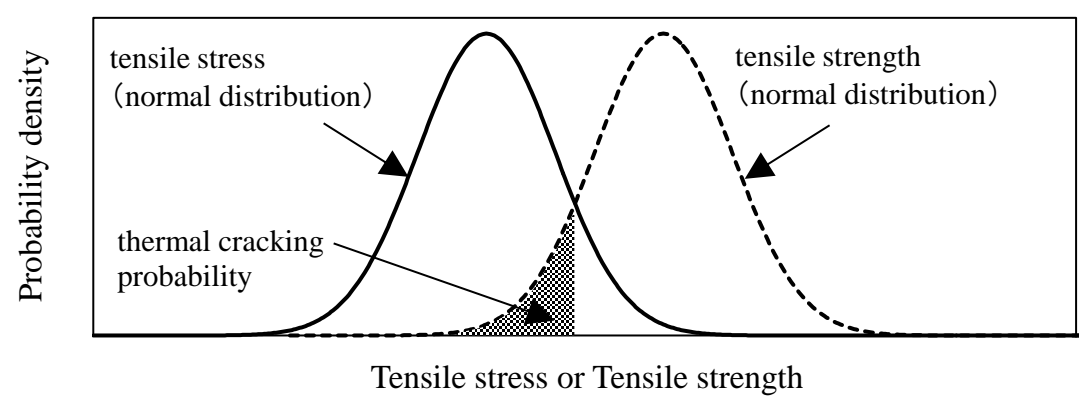

Figure 3. Outline of R-S model

\section{OCCURRENCES OF THERMAL CRACKING}

Out of a total of 130 sections, occurrences of thermal cracking were observed in 64 sections, and were not observed in 66 sections. Therefore, the overall rate of thermal cracking was $49.2 \%$. 
Figure 4 shows the histograms of the slump, compressive strength (material age of 28 days), and concrete temperature at the time of placement, in terms of the presence or absence of thermal cracking. The average slump value is $9.7 \mathrm{~cm}$ in the presence of cracking and $9.9 \mathrm{~cm}$ without, which are comparable. However, the slump in the presence of cracking is generally smaller. The average value of the compressive strength is $42.9 \mathrm{~N} / \mathrm{mm}^{2}$ in the presence of cracking and $44.0 \mathrm{~N} / \mathrm{mm}^{2}$ without cracking. A trend is seen in that the compressive strength has slightly smaller values and a greater variation in the presence of cracking. The coefficient of variation of the overall compressive strength is $6.1 \%$, which is smaller than that of ordinary ready-mixed concrete. The average concrete temperature at the time of placement is $22.7^{\circ} \mathrm{C}$ in the presence of cracking and $19.6^{\circ} \mathrm{C}$ without cracking. Thus, a trend is seen in greater values in the presence of cracking. This difference in concrete temperature would be due to the difference in the period of construction, and the slightly smaller values in compressive strength in the presence of cracking would be due to the seasonal variation.

Thus, the greater the compressive strength, the greater the variation, and the higher the concrete temperature, the more thermal cracking tends to occur, which seems to be a reasonable result.

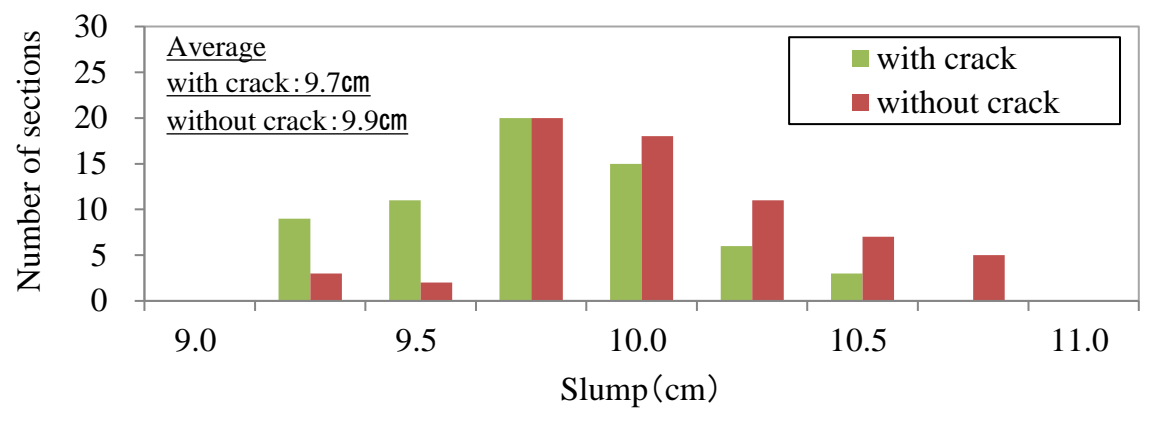

(Slump)

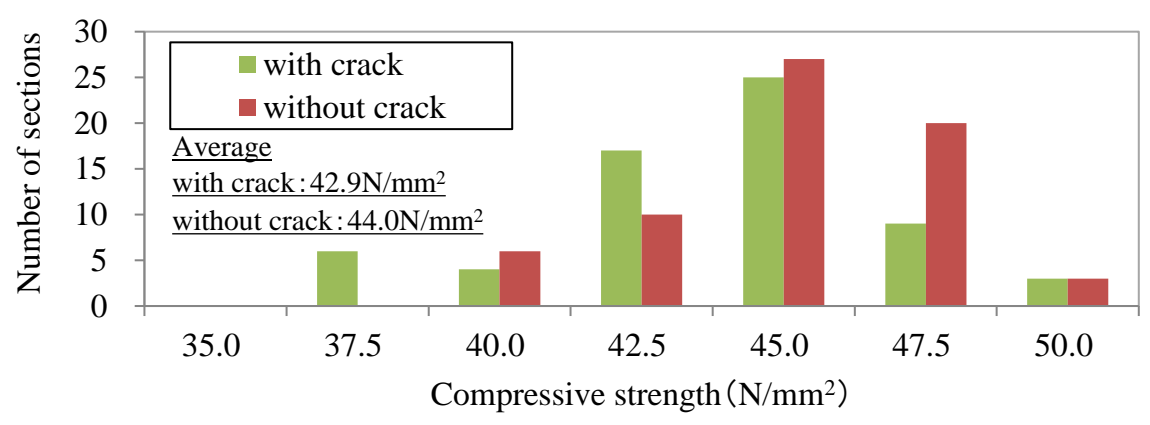

(Compressive strength) 


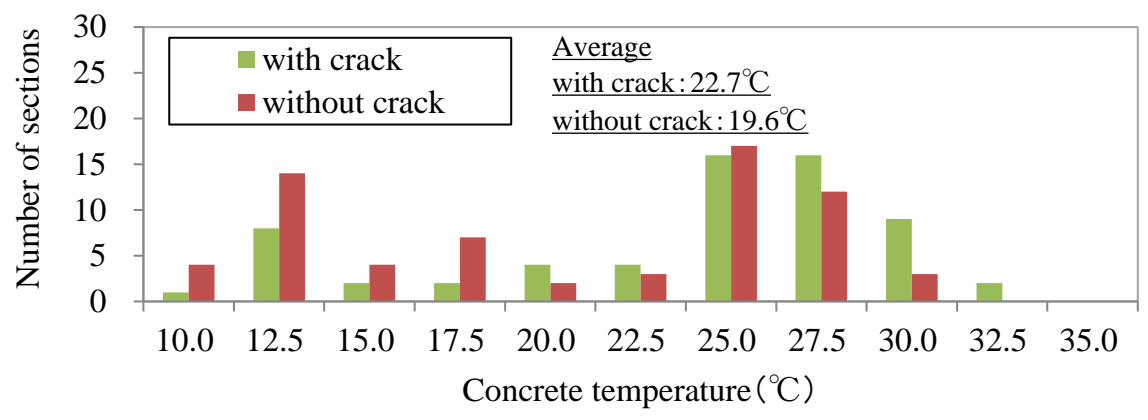

(Concrete temperature at the time of placement)

Figure 4. Histograms of slump, compressive strength, and concrete temperature

\section{EVALUATION RESULTS}

\section{Results of Thermal Stress Analysis}

Figure 5 shows a histogram in the case where the thermal cracking indexes are segmented by 0.05 in terms of presence or absence of thermal cracking. The average value of the thermal cracking index is 1.07 in the presence of thermal cracking and 1.10 without cracking, which are comparable. The thermal cracking indexes are distributed in the range of $0.80-1.20$ in the presence of thermal cracking and 0.851.35 without cracking. Thus, the thermal cracking indexes without cracking are generally distributed in a greater range.

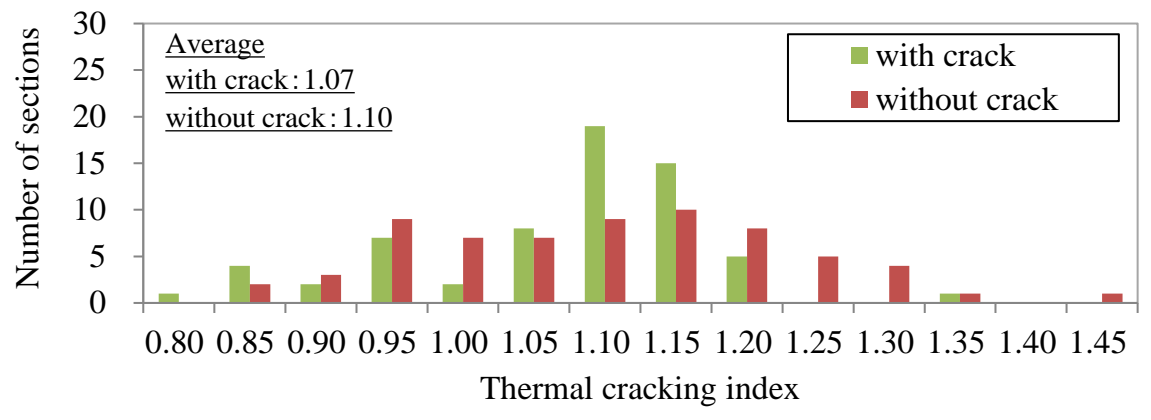

Figure 5. Histogram of thermal cracking indexes

Figure 6 shows the histograms of tensile stress and tensile strength corresponding to the minimum thermal cracking indexes in terms of the presence or absence of thermal cracking. The average value of the tensile stress is $2.99 \mathrm{~N} / \mathrm{mm}^{2}$ in the presence of cracking and $2.86 \mathrm{~N} / \mathrm{mm}^{2}$ without cracking, which are comparable. The average value of the tensile strength is $3.16 \mathrm{~N} / \mathrm{mm}^{2}$ in the presence of cracking and $3.11 \mathrm{~N} / \mathrm{mm}^{2}$ without cracking, which are also comparable. However, the tensile strength values without cracking are distributed in a larger range. It is presumed that this is the factor in which the thermal cracking indexes without cracking are generally distributed in a greater range.

The overall coefficients of variation of the tensile stress and tensile strength are $13.4 \%$ 
and $7.4 \%$, respectively. In thermal stress analyses, the tensile strength is obtained from the relationship with compressive strength. Further, Young's modulus that is used for calculating the tensile stress is also obtained from the relationship with compressive strength. Therefore, it is naturally presumed that the coefficients of variation of the compressive strength would be comparable with those of the tensile stress and tensile strength. However, the results show greater coefficients of variation of the tensile stress and tensile strength compared to that of the compressive strength, $6.1 \%$, and this trend is more significant in the tensile stress. It is presumed that the coefficient of variation of the tensile strength resulted in a greater value than that of the compressive strength because compressive strength tends to be more affected by the difference in concrete temperature, member thickness, and placement period. On the other hand, in the case of calculating the tensile stress, not only the concrete temperature and placement period but also the member size of each side wall and timing for arranging construction joints add effects with different constraint conditions, and the variation factor would increase. It is presumed that this is the reason why the coefficient of variation of the tensile stress became significantly greater than that of the compressive strength.

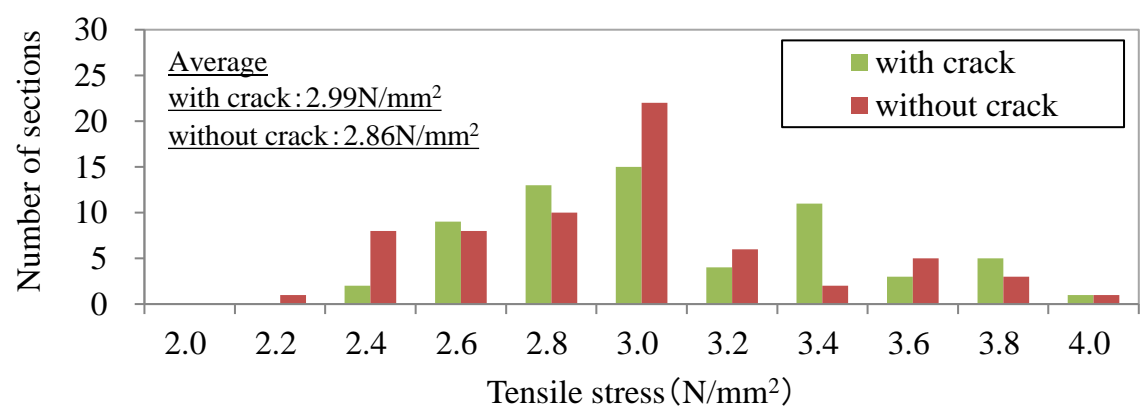

(Tensile stress)

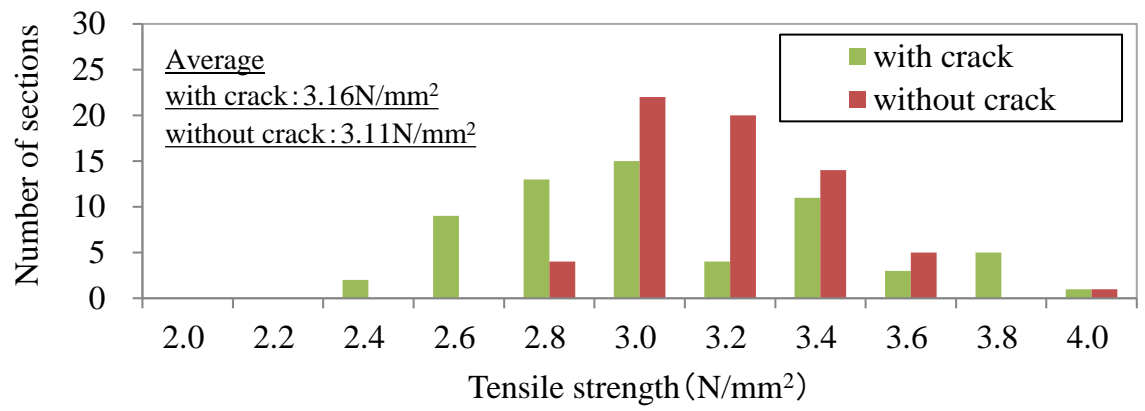

(Tensile strength)

Figure 6. Histograms of tensile stress and tensile strength

Relationship between thermal cracking index and thermal cracking probability. Figure 7 shows the actual thermal cracking rates divided by 0.05 of the thermal cracking index. The figure shows the relationship between the thermal cracking index and thermal cracking probability shown in the JCI Guidelines as well as the relationship between the thermal cracking index and thermal cracking probability 
stochastically calculated using the R-S model. As seen in Figure 5, the data for the thermal cracking index of 0.80 and $1.35-1.45$ is so sparse, 0 to 2 sections, that it has been omitted.

The actual rate of thermal cracking tends to decrease as the thermal cracking index increases. However, considerably different values of the thermal cracking indexes were observed for the same rate of thermal cracking; for example, thermal cracking indexes of $0.90-1.20$ were observed for a thermal cracking rate of about $40 \%$.

The relationship between the thermal cracking index and thermal cracking probability calculated using the R-S model shows a trend that the gradient of the curve is steeper than that of the JCI Guideline; for example, the thermal cracking index is 0.90 for a thermal cracking probability of about $80 \%$, and the index is 1.10 for a probability of about $30 \%$. Thus, when the coefficients of variation of the tensile stress and tensile strength are determined, the relationship between the thermal cracking index and thermal cracking probability should be uniquely determined from a stochastic viewpoint. However, the actual occurrences of thermal cracking show a certain range of the thermal cracking index for the same probability, that is, some separation is found between them. This can be attributed to various factors, but it might be due to the material heterogeneity of the members. Although the properties of a member are applied as being homogeneous in thermal stress analyses, it is not necessarily so in actual construction due to the influence of material segregation, etc. Depending on the influence of material segregation, the actual tensile stress or tensile strength values may be larger or smaller than the analysis results, which may affect the thermal cracking indexes.

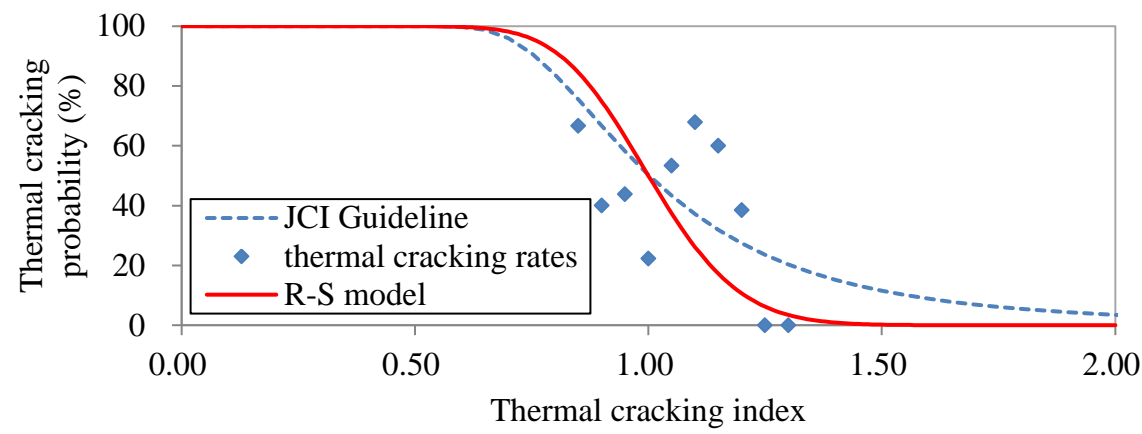

Figure 7. Relationship between thermal cracking index and thermal cracking probability

Figure 8 shows the shifts of the R-S model in the left and right directions so that they match the actual rates of thermal cracking, assuming that the thermal cracking indexes change due to the influence of homogeneity. The figure shows that the two curves obtained by multiplying the R-S model by 0.9 and 1.15 closely match the actual thermal cracking rates. 


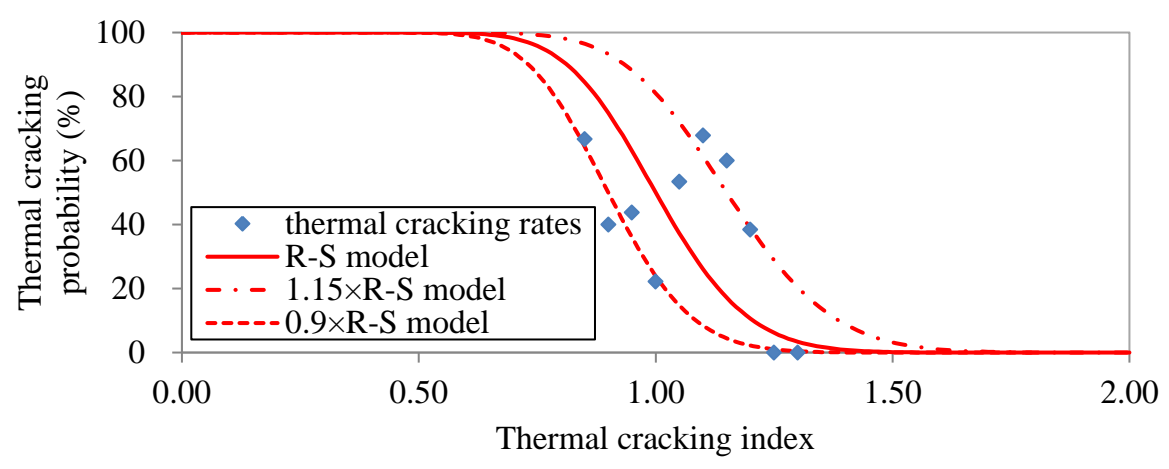

Figure 8. Relationship between thermal cracking index and thermal cracking probability

The tensile strength estimation formula of the JCI Guidelines used in the thermal stress analysis is based on the lower limit values of various splitting tensile strength tests, and it has been reported in previous studies that the tensile strength obtained from the formula is about 0.93 times the actual thermal cracking occurrence strength [Ajiti et al. 2013]. Therefore, it is presumed that the structures that match the thermal cracking probability curve by multiplying the R-S model by 0.9 were homogeneous and their tensile strength was about $10 \%$ greater than that in the analysis. On the other hand, it is also presumed that the structures that match the thermal cracking probability curve by multiplying the R-S model by 1.15 were not so homogeneous and their tensile strength was less than that in the analysis and generated greater tensile stress. Previous studies have shown that the greater the amount of coarse aggregate, the lower the compressive strength, and they have a linear relationship [Kawakami, 1969]. The greater the amount of coarse aggregate in the lower part of the side wall under greater restraining conditions, the lower the tensile strength due to the decrease in compressive strength. Since temperature stress has a small stress region, even a minor material segregation that does not affect the strength of the actual structure may have an effect on the thermal cracking. In addition, when a structure is not homogeneous it may be prone to generating cracks due to locally weakened parts.

Although they were constructed under the same conditions of concrete mix proportion and the same construction method, the target side wall structures in this study have been grouped into two thermal cracking probability curves presumably due to the generation of different homogeneities in the actual structures.

Comparing the structures that match the curve for 0.9 times the R-S model and those that match the curve for 1.15 times, the average slump values are 9.8 and $9.9 \mathrm{~cm}$, respectively, which are comparable. The average compressive strength values are 43.0 and $43.2 \mathrm{~N} / \mathrm{mm}^{2}$, respectively, which are also comparable. Thus, the difference in the slump or compressive strength does not have a recognizable effect on the homogeneity. The average concrete temperatures at the time of placement are $20.6^{\circ} \mathrm{C}$ and $23.4^{\circ} \mathrm{C}$, respectively, that is, the latter tends to have been constructed at the time of higher temperature. The average wall thickness values are 1.46 and $1.22 \mathrm{~m}$, respectively, that is, the latter tends to be thinner. Although this study has not clarified the effects of the 
difference in these tendencies on the homogeneity, it may be possible that differences in environmental or structural conditions at the time of construction influenced the homogeneity.

From the above, it may be possible to set the relationship between the actual thermal cracking indexes and thermal cracking probabilities based on the R-S model, by correcting the thermal cracking indexes related to the homogeneity of the actual structures. As the correction rates for the thermal cracking indexes, 0.87 (1/1.15) to $1.11(1 / 0.9)$ have been set in this study.

\section{CONCLUSION}

In this study, thermal stress analyses were conducted on multiple side walls constructed under the same conditions of concrete mix proportion and the same construction method, and the rates of thermal cracking in the structures were obtained from the actual occurrences of thermal cracking. In addition, the relationship between the thermal cracking indexes and thermal cracking probabilities were stochastically obtained using the R-S model. Based on these results, the factors that affect the thermal cracking probability as well as their degree of influence were studied. The findings obtained from this study are as follows:

(1) As a result of thermal stress analyses, the coefficients of variation of tensile stress and tensile strength were obtained as $13.4 \%$ and $7.4 \%$, respectively. These are greater than that of the compressive strength, $6.1 \%$, and that of the tensile stress was especially greater.

(2) Regarding the thermal cracking probabilities from the R-S model, different thermal cracking indexes were obtained for the same rate of thermal cracking. This result is thought to be due to the effects of the homogeneity of the structures. Considering the homogeneity of the structures for correcting the thermal cracking index, correction rates of $0.87-1.11$ were obtained in this study.

Going forward, it is necessary to understand the factors that affect the homogeneity of structures, as well as to conduct experimental and analytical studies to reveal how they affect the thermal cracking probabilities.

\section{REFERENCES}

Ajiti, T., Arai, J., Senba, R., and Mizobuchi, T. (2013). "A Consideration on Design Values Used for Temperature Cracked Inspection." Proceedings of the Japan Concrete Institute, 35, 1285-1290.

Japan Concrete Institute (2016), "The Guidelines for Control of Cracking of Mass Concrete 2016."

Japan Society of Civil Engineers (2017), "Standard Specifications for Concrete Structures-2017."

Kawakami, H. (1969), "Effects of Size and Quantity of Aggregate on Compressive Strength of Concrete (1)." Transactions of the Architectural Institute of Japan, 
$166,19-27$.

Nakamura, H., and Hamada, S. (1995). "Analytical Studies on The Cracking Probabilities for The Mass Concrete Structures Having Unceration Properties." Journal of JSCE, 514, 29-40.

Sugihashi, N. (2017), "Studies on the Present Issues and Improvements of Checking Methods for Thermal Cracking Based on Their Histories." Doctoral dissertation. 